\title{
Synthesis of photoluminescent-doped poly(methyl methacrylate)
}

\author{
Material used in POF technology
}

\author{
Małgorzata Gil ${ }^{1}$ (D) Beata Podkościelna ${ }^{2} \cdot$ Paweł Mergo $^{1} \cdot$ Barbara Gawdzik $^{2}$
}

Received: 13 November 2018/ Accepted: 22 March 2019/Published online: 5 April 2019

(c) The Author(s) 2019

\begin{abstract}
Synthesis of optical materials, copolymers based on methyl methacrylate used as a polymer matrix, and 2,7-bis[2-(2hydroxy-3-methacryloyloxypropoxy)]naphthalene (2,7-NAF.DM) applied as a photoluminescent dopant are presented. The purification procedure as well as the detailed information about the method of synthesis is shown. Additionally, the effect of varying concentrations of dopant to determine its effect on the course of polymerization process and thermal properties was tested. Possible application of the received materials as optical materials has been studied by the use of the coupled thermal and spectroscopic methods (TG/DTG/QMS). Using these methods, in addition to quantitative information (mass change, process heat), the qualitative information regarding the chemical composition of the gas products is obtained.
\end{abstract}

Keywords Optical fibers · Thermogravimetry $\cdot$ Spectroscopy $\cdot$ Polymer $\cdot$ Photoluminescent dopant

\section{Introduction}

Photoluminescent monomers and polymers are contemporary and leading materials, which find applications in the rapidly developing areas of the industry as optoelectronics or photonics [1-7]. These unique materials may be used, for example, as organic projectors and for construction of energy-saving light source [8]. Therefore, demand for new and more resistant materials is still increasing, becoming a challenge for researchers. Materials so far used in photoluminescence, e.g., zinc sulfide with copper and cobalt admixture or inorganic aluminum compounds, have been changed to reactive and more accessible organic compounds [9-12].

To use polymeric materials in photonics, it is necessary to adhere to the appropriate temperature. Exceeding it, one

Małgorzata Gil

malgorzata.gil@poczta.umcs.lublin.pl

1 Laboratory of Optical Fibers Technology, Maria CurieSklodowska University, M. Curie-Sklodowska Sq. 5, 20-031 Lublin, Poland

2 Department of Polymer Chemistry, Faculty of Chemistry, Maria Curie-Sklodowska University, M. Curie-Sklodowska Sq. 5, 20-031 Lublin, Poland can change the properties of the material or even lead to its thermal decomposition. In the case of the highest quality optical fibers, always the mass loss is the most crucial value, because drawing a good optical fibers becomes problematic. It was investigated that the value is about 5 mass $\%$ of mass loss, enable for successive drawing process [13]. Nowadays, poly(methyl methacrylate) (poly(MMA)) is often used as polymeric material in optical fiber technology. To improve its optical properties, dopant compounds are used. Doped material is suitable as the active coating on the optical fibers and as the active core material for the polymer optical fibers. Such compounds have influence not only on optical but also on thermal and mechanical properties of both preforms and final fiber. Typical organic dopants used in optical fibers technology are: dye-rhodamine [14-16], dye-fluorescein [17, 18] or rare-earth organic complexes $[19,20]$.

In this article, studies' materials used in polymer optical fibers technology are presented. As matrix material poly(methyl methacrylate) was used, whereas dimethacrylate derivative of naphthalate-2,7-diol (2,7-NAF.DM) was applied as photoluminescent dopant.

The novelty of our research is the application of a photoluminescent dopant (2,7-bis[2-(2-hydroxy-3methacryloyloxypropoxy)]naphthalene) in the character of 
a polymerizable monomer for the synthesis of polymeric optical fiber materials. 2,7-NAF.DM contains vinyl bonds in its structure, which can react with methyl methacrylate as a result of polymerization reaction. Consequently, the much stronger system is formed comparing to those containing, for example, rhodamine or fluorescein, which is only physically suspended/dissolved in poly(MMA).

During the tests, the effect of different concentrations of dopant was studied to determine its effect on the course of the polymerization process and, thus, the thermal properties of the obtained copolymers. The synthesized materials can be used to obtain photoluminescent polymeric optical fibers.

\section{Experimental}

\section{Materials}

Methyl methacrylate, thioglycolic acid (98\%) and dibenzoyl peroxide were purchased from Sigma-Aldrich. The detailed information about the synthesis of 2,7-(2-hydroxy3-methacryloyloxypropoxy)naphthalene (2,7-NAF.DM) was presented in Ref. [21, 22]. The 2,7-NAF.DM was obtained in the two-step reaction. In the first step, naphthalene-2,7-diol (2,7.NAF) was reacted with epichlorohydrin. Next, esterification of the obtained diglycidyl ether was carried out with the use of methacrylic acid in the presence of hydroquinone (polymerization inhibitor) and triethylbenzylammonium chloride as a catalyst. The chemical structure of 2,7-NAF.DM is presented in Fig. 1.

\section{Purification and polymerization procedure}

A very important issue in the production of materials for optical applications is their purity. Therefore, in order to eliminate the effects of unknown impurities, which definitely would increase the scattering of light, and thus will<smiles>C=C(C)C(=O)OC</smiles>

(a) MA (b)<smiles>O=C(O)CS</smiles>
(c)<smiles>O=C(OOC(=O)c1ccccc1)c1ccccc1</smiles>
$\mathrm{OH}$<smiles>C=C(C)C(=O)OCC(O)COc1ccc2ccc(OCC(O)COC(=O)C(=C)C)cc2c1</smiles>

2,7-NAF.DM

Fig. 1 Chemical structures of the compounds used in thermal polymerization a methyl methacrylate, $\mathbf{b}$ thioglycolic acid, $\mathbf{c}$ benzoyl peroxide and d 2,7-NAF.DM also increase the attenuation, MMA was purified before starting the process of thermal polymerization. The purification was performed by distillation under pressure conditions getting rid of impurities as well as undesirable inhibitor. The initiator dibenzoyl peroxide, which was also purified before the use, and chain transfer agent thioglycolic acid were used in thermal free radical polymerization of examined samples. Chemical structures of the methyl methacrylate, thioglycolic acid and benzoyl peroxide are presented in Fig. 1. In optical fibers technology, two methods of polymerization are used-thermal and photopolymerization (the process, essentially, takes place in the same way, the main difference is the source of initiation). In this study, the thermal polymerization process took place. The main reason for our choice was that photopolymerization causes cross-linking of the polymer, and only polymers with a linear structure can be subjected to the drawing process. Another problem is the thickness of the sample obtained by photopolymerization. Unfortunately, due to the limited penetration of UV light, using this method it is possible to obtain samples only of a thickness of $2-3 \mathrm{~mm}[23,24]$. After mixing the mentioned ingredients, all the compositions were prepolymerized, and then the appropriate curing process applied. The thermal polymerization was carried out in a test tube with an inner diameter of $14 \mathrm{~mm}$. The process was carried out in an oven under vacuum. The temperature was increased gradually from 25 to $110{ }^{\circ} \mathrm{C}$ in 2 days until solidification was fulfilled. All measurements were performed in an inert gas atmosphere, because oxygen is a radical scavenger by which the free radical polymerizations can be terminated. The same atmosphere was used during the polymer optical fiber drawing process.

The series of compositions with MMA as the basic component, dimethacrylate derivative of naphthalate-2,7diol as dopant, benzoyl peroxide as an initiator and thioglycolic acid as the chain transfer agent are obtained according to amount presented in Table 1. In Fig. 2,

Table 1 Experimental parameters of the syntheses

\begin{tabular}{lllll}
\hline Sample & \multicolumn{3}{l}{ Composition/mass\% } \\
\cline { 2 - 5 } & MMA & Dopant & Initiator & Chain transfer agent \\
\hline Sample no. 1 & 99.9 & 0.1 & 0.4 & 0.8 \\
Sample no. 2 & 99.8 & 0.2 & 0.4 & 0.8 \\
Sample no. 3 & 99.5 & 0.5 & 0.4 & 0.8 \\
Sample no. 4 & 99.3 & 0.7 & 0.4 & 0.8 \\
Sample no. 5 & 99.1 & 0.9 & 0.4 & 0.8 \\
Sample no. 6 & - & 100 & 0.4 & 0.8 \\
Sample no. 7 & 100 & - & 0.4 & 0.8 \\
\hline
\end{tabular}




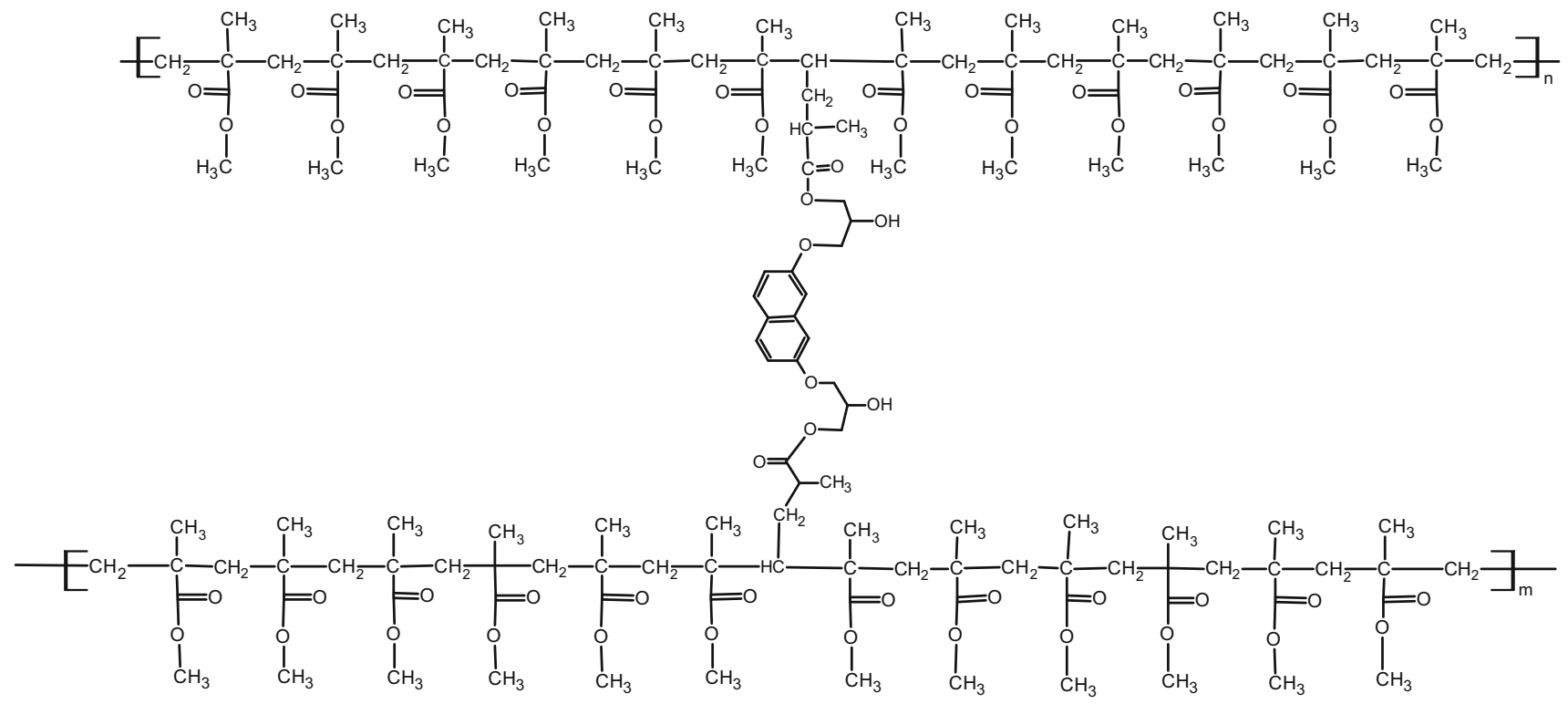

MMA-co-2,7-NAF.DM

Fig. 2 Fragment of proposal structure of the obtained copolymers

probable fragment of structure of the obtained copolymers is shown. Photoluminescent monomer 2,7-NAF.DM possesses in their chemical structure two double bonds, and during copolymerization reaction low cross-linking effect is possible. But, due to the fact that its addition is small $(0.1-0.9 \% \mathrm{w} / \mathrm{w})$, it does not affect further mechanical processing of received polymers.

\section{Characterization}

Attenuated total reflection-Fourier transform infrared (ATR/FTIR) spectra were obtained using a Bruker FTIR spectrophotometer TENSOR 27 (Bruker, Germany). Spectra were recorded in the frequency range of $600-4000 \mathrm{~cm}^{-1}$ with a resolution of $4 \mathrm{~cm}^{-1}$ and 32 scans.

Room temperature UV-Vis absorption spectra were recorded using a V-660 JASCO spectrophotometer.

Thermal analysis was carried out on a STA 449 Jupiter F1, Netzsch (Selb, Germany), under the following operational conditions: heating rate $10{ }^{\circ} \mathrm{C} \mathrm{min}^{-1}$, the dynamic atmosphere of helium $\left(50 \mathrm{~mL} \mathrm{~min}{ }^{-1}\right)$ in the temperature range of $30-600{ }^{\circ} \mathrm{C}$, sample mass of about $5 \mathrm{mg}$, sensor thermocouple type $\mathrm{S}$ TG-DTA. As a reference, empty $\mathrm{Al}_{2} \mathrm{O}_{3}$ crucible was used. The volatile products coming out during depolymerization and/or decomposition process were detected by quadrupole mass spectrometer (QMS) 403C Aëolos (Germany) coupled on-line to STA instrument. The mass spectrometer was connected on-line to STA instrument by quartz capillary heated to $300{ }^{\circ} \mathrm{C}$. The QMS was operated with an electron impact ionizer with energy $70 \mathrm{eV}$. The measurements performed in scan mode for $m / z$, where $m$ is the mass of molecule and $z$ is a charge of the molecule in electron charge units in the range from 10 to $110 \mathrm{amu}$, allowed to identify all possible volatile particles produced during the decomposition.

\section{Results and discussion}

\section{Spectroscopic characterization of the materials}

In Fig. 3, the ATR-FTIR spectra of the MMA-co-2,7NAF.DM (methyl methacrylate-co-2,7-bis[2-(2-hydroxy-3methacryloyloxypropoxy)]naphthalene) copolymers are presented. Additionally, homopolymer MMA for

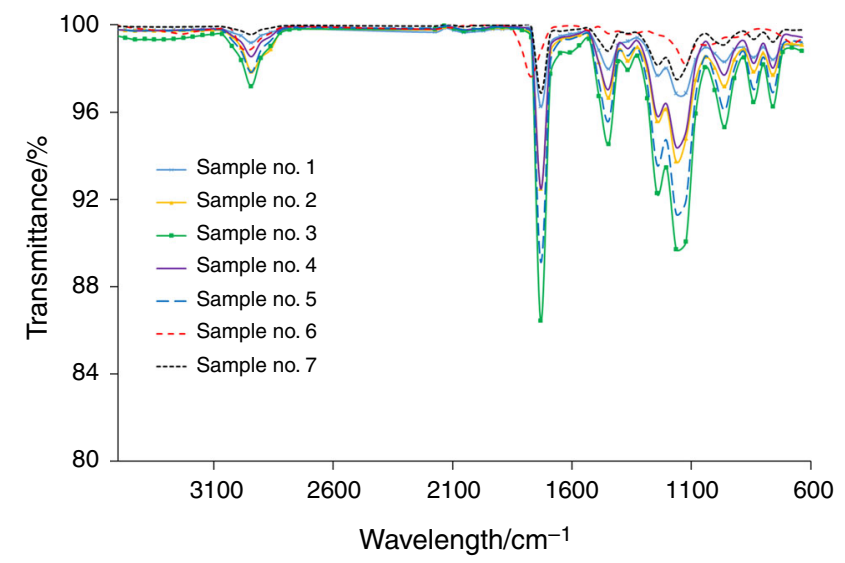

Fig. 3 ATR-FTIR spectra of the synthesized materials 
comparison purposes was added. Because the main components of all copolymers are MMA, the course of all spectra is similar and typical for methacrylate derivatives. Only the small changes in the intensity of shape are noticeable. In the spectra of all derivatives, $\mathrm{C}-\mathrm{H}$ stretching vibrations of aromatic ring backbone methylene and methyl groups were observed at the range $2994-2852 \mathrm{~cm}^{-1}$. The strong signal of the $\mathrm{C}=\mathrm{O}$ group occurred at $1724 \mathrm{~cm}^{-1}$; additionally, the signal of the $-\mathrm{C}-$ $\mathrm{O}-\mathrm{C}-$ group is observed at $1250 \mathrm{~cm}^{-1}$. For all copolymers, absorption of aromatic naphthalene $(\mathrm{C}-\mathrm{H})$ was observed in about $842 \mathrm{~cm}^{-1}$ [22].

UV-Vis absorption spectra of the copolymers and monomer solutions are shown in Fig. 4. We can see that the strong absorption in the UV region $(250-360 \mathrm{~nm})$ obviously originates from naphthalene [25]. On the spectrum, we can also note that the band $450 \mathrm{~nm}$ originates

(a) Monomer solution:

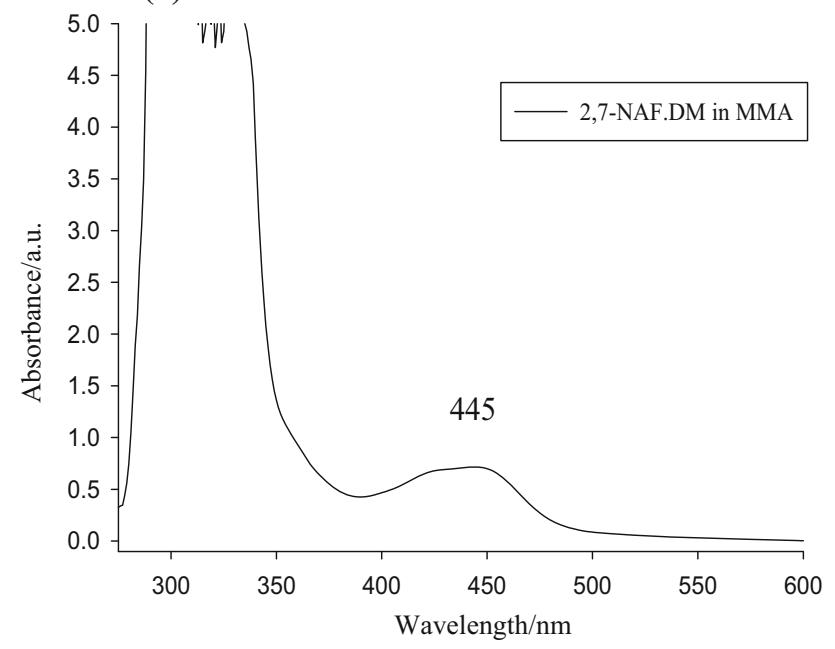

(b) Copolymer:

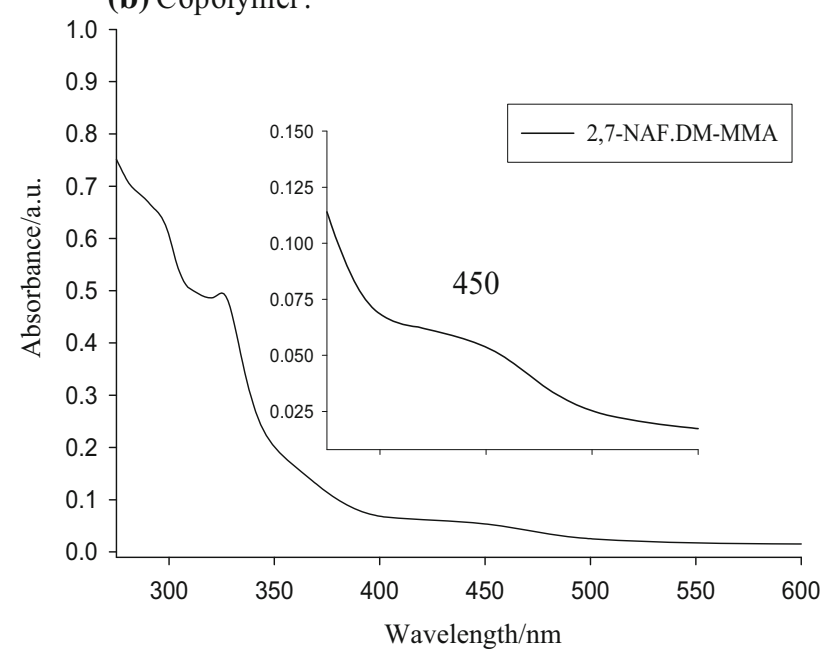

Fig. 4 Absorption spectra of 2,7-NAF.DM with MMA in solutions (a) and copolymers (b) from the $\pi$ conjugation in the naphthalenediepoxymethacrylate moiety. This absorption is strongly connected with the position of substituent in naphthalene $(2,7)$. This phenomenon was described in detail previously [22, 25]. After excitation by UV radiation, the sample emits yellow-green radiation, which coincides with the range of wavelength for green light (500-542 nm) and for yellow one (542-578 nm). Figure 5 shows the photograph of the polymer samples which were exposed to UV light (2 UV lamps $2 \times 4 \mathrm{~W}$ ). It is clearly visible the photoluminescence effect of polymeric preforms. This phenomenon becomes stronger with the increase of 2,7-NAF.DM amounts.

\section{Thermogravimetric and spectroscopic analysis}

Among the several techniques used for the investigation of thermal characteristics of polymer, only the coupled thermal and spectroscopic methods can get, in addition to quantitative information such as mass change or process heat, the qualitative information regarding the chemical composition of the gas products.

The use of coupled thermal (TG/DTG) and spectroscopic (MS) methods allowed for a complete analysis of the tested samples. Based on thermogravimetric data (Figs. 6, 7), it was possible to determine the mass loss at which the samples decomposition or depolymerization begins and the mass loss at the temperature at which polymer optical fibers are drawn. These two parameters are crucial in optical fibers technology. The temperature at which optical fibers are drawn determines the main component-the polymer matrix; in this case, it is poly(methyl methacrylate). Poly(MMA) thus obtained by us is characterized by a very good thermal resistance. It is thermally stable up to $280^{\circ} \mathrm{C}$, and that means that till this temperature mass loss is not observed. For any polymer optical fibers (POF) which would be drawn using this material, $280{ }^{\circ} \mathrm{C}$ is the temperature limit, above which good optical and mechanical properties cannot be achieved. Typically, the fiber drawing process is carried out at a temperature of about $220^{\circ} \mathrm{C}$, in which the poly(MMA) is sufficiently malleable. Any addition

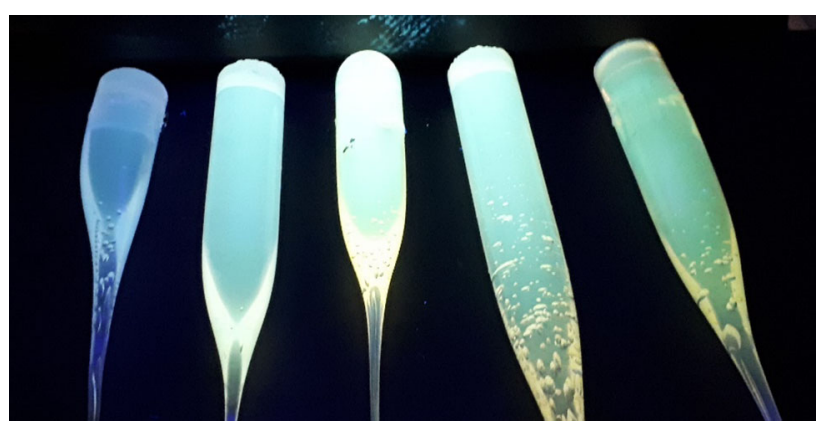

Fig. 5 Photographs of the materials with photoluminescent dopant 


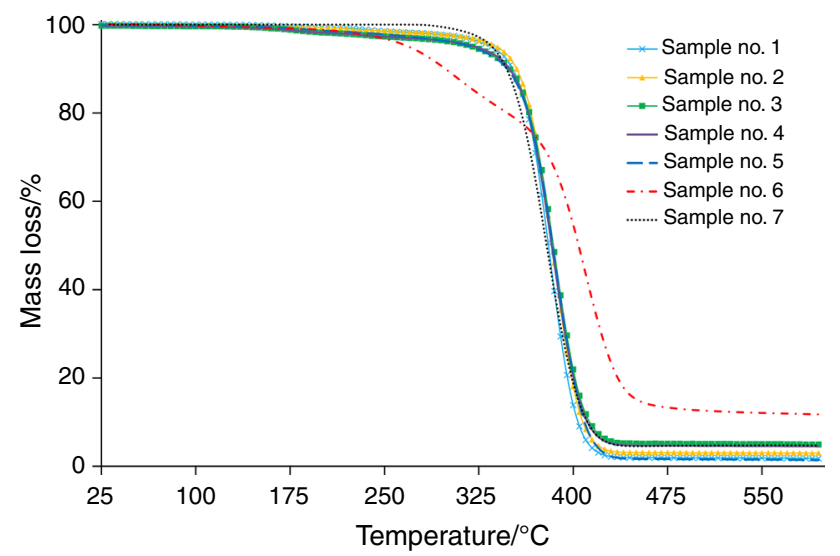

Fig. 6 TG curves for all samples

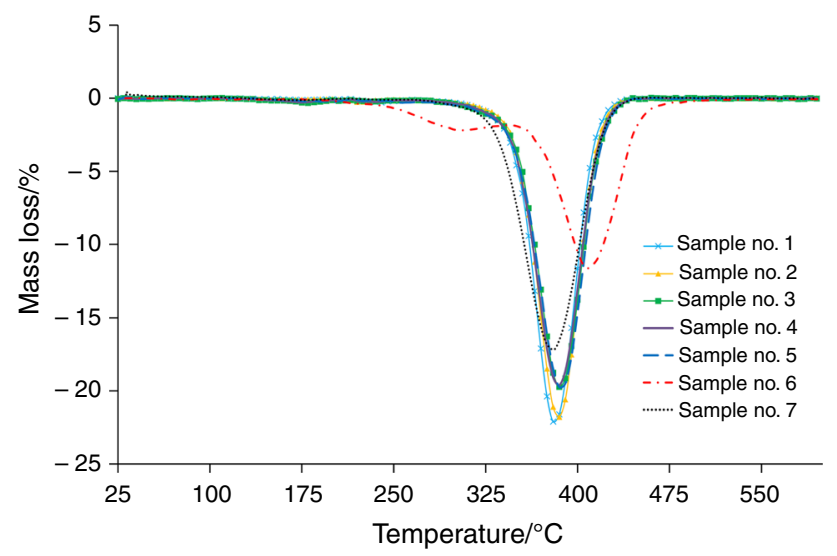

Fig. 7 DTG curves for all samples

of another substance will result in changes in the parameters of the optical fiber drawing process (e.g., fiber drawing speed, temperature of the oven). The applied photoluminescence dopant exhibits good thermal properties and is stable up to $195{ }^{\circ} \mathrm{C}$, but it should be noted that at $220{ }^{\circ} \mathrm{C}$ it showed a mass loss of 1.8 mass $\%$.

All the thermoanalytical results-the proper decomposition temperature and mass loss at this temperature, the maximum temperature of decomposition, the mass loss at $220{ }^{\circ} \mathrm{C}$ and residual mass-are summarized in Table 2 , and in Table 3 the percentage of mass loss (in the range 1-5 mass\%) and the temperature in which the mass loss occurred were placed.

Samples no. 1-5 contain 0.1-0.9 mass\% of photoluminescent dopant. For comparison purposes, samples no. 6 and 7 were synthesized. Sample no. 6 is formed from pure poly(2,7-NAF.DM), whereas sample no. 7 is in the form of homopolymer of poly(MMA).

Based on the data collected in Table 2, one can see that proper (initial) decomposition temperatures vary in the range of $220^{\circ} \mathrm{C}$, for sample with the highest dopant concentration (sample no. 5), to $250{ }^{\circ} \mathrm{C}$, for samples with the lowest dopant concentration (samples no. 1 and 2). Comparing the amount of mass loss at these temperatures, for samples no. 1 and 2, this value is about 1.4-1.5 mass \% and for samples no. 2-5 varies in the range of 1.8-2.6 mass\%, we can obviously conclude that as the content of the dopant increases, the polymerization process is more disturbed, which results in the deterioration of the thermal properties of the pure matrix. As it was already mentioned, one of the most important parameter taken into account when choosing a polymer material that could be used in drawing process is its thermal stability at the temperature of the process itself $\left(220^{\circ} \mathrm{C}\right)$. It can be concluded based on the data collected in Table 2 that the samples with dopant concentration 0.1 and 0.2 mass\% (samples no. 1 and 2) have the smallest amount of mass loss at this temperature. The highest mass loss was observed for samples no. 3 and 4, where the value is about 2.1 mass\%; for sample no. 5 ( 0.9 mass\% dopant), the value is similar as for pure photoluminescent dopant (sample no. 6) and is 1.7-1.8 mass\%. The temperature of the maximum decomposition is almost the same for all tested samples (varies in the range $380-390{ }^{\circ} \mathrm{C}$ ); only for sample no. 6 , this temperature is much more lower $\left(302{ }^{\circ} \mathrm{C}\right)$. Also, the residual mass is about the same for all samples and varies in the range of 1.8-5.0 mass\%; only for pure dopant, this value is about

Table 2 Thermal stabilities of all samples

\begin{tabular}{|c|c|c|c|c|c|c|c|}
\hline & $\begin{array}{l}\text { Sample } \\
\text { no. } 1\end{array}$ & $\begin{array}{l}\text { Sample } \\
\text { no. } 2\end{array}$ & $\begin{array}{l}\text { Sample } \\
\text { no. } 3\end{array}$ & $\begin{array}{l}\text { Sample } \\
\text { no. } 4\end{array}$ & $\begin{array}{l}\text { Sample } \\
\text { no. } 5\end{array}$ & $\begin{array}{l}\text { Sample } \\
\text { no. } 6\end{array}$ & $\begin{array}{l}\text { Sample } \\
\text { no. } 7\end{array}$ \\
\hline Mass loss at $220^{\circ} \mathrm{C} /$ mass $\%$ & 0.8 & 0.9 & 2.1 & 2.1 & 1.7 & 1.8 & 0 \\
\hline Initial decomposition temperature $/{ }^{\circ} \mathrm{C}$ & 245 & 250 & 240 & 240 & 220 & 195 & 280 \\
\hline $\begin{array}{l}\text { Mass loss at proper (initial) decomposition } \\
\text { temperature/mass } \%\end{array}$ & 1.5 & 1.4 & 2.6 & 2.6 & 1.8 & 1.1 & 0.0 \\
\hline DTG $\max { }^{\mathrm{a}} /{ }^{\circ} \mathrm{C}$ & 380 & 385 & 385 & 385 & 390 & 302 & 380 \\
\hline Residual mass/mass\% & 1.8 & 3.0 & 5.0 & 4.6 & 1.5 & 11.8 & 5.0 \\
\hline
\end{tabular}

${ }^{a}$ DTG max, first observed maximum 
Table 3 Determination of mass loss temperature from 1 to 5 mass $\%$

\begin{tabular}{llllll}
\hline Sample number & \multicolumn{5}{l}{ Mass loss/mass\% } \\
\cline { 2 - 6 } & 1 & 2 & 3 & 4 & 5 \\
\hline 1 & 231 & 283 & 314 & 327 & 335 \\
2 & 223 & 292 & 319 & 332 & 340 \\
3 & 170 & 216 & 270 & 306 & 320 \\
4 & 165 & 214 & 267 & 306 & 322 \\
5 & 185 & 241 & 279 & 304 & 319 \\
6 & 190 & 227 & 248 & 260 & 270 \\
7 & 306 & 321 & 329 & 335 & 339 \\
\hline
\end{tabular}

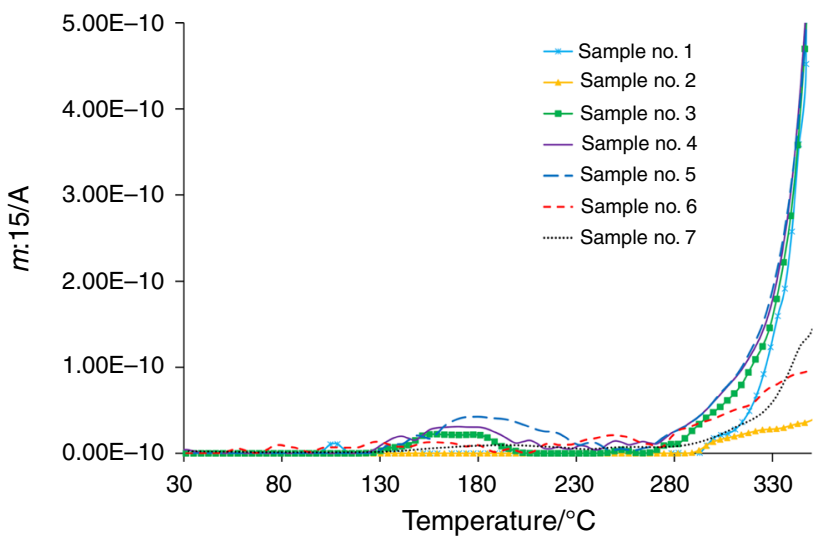

Fig. 8 Mass spectroscopy curves for ion 15

12 mass \%. Thermoanalytical studies of pure poly(MMA) matrix, as it was already mentioned, indicate no mass loss up to the initial decomposition temperature. Based on the data collected in Table 3, it can be seen that the addition of photoluminescent compound deteriorates this property. For the mixture containing 0.1 and 0.2 mass\% (samples no. 1 and 2) of the dopant, the first mass loss (1 mass\%) is observed at a temperature range of about $220-230{ }^{\circ} \mathrm{C}$, while for the samples no. 2-5 (0.5, 0.7 and 0.9 mass $\%$ dopant) the temperature for the same mass loss drops to the range $170-185{ }^{\circ} \mathrm{C}$. Therefore, it can be concluded that samples no. 1 and 2 only slightly change the thermal stability of the poly(MMA).

Based on the results obtained from mass spectroscopy, the presence of two ions, which can illustrate the decomposition, was detected (Figs. 8, 9) [26]. Ion $15 \mathrm{~m} / \mathrm{z}$ most likely is responsible for the presence of methyl groups (Fig. 8). As one can see that this ion, for samples no. 1, 2 and 7 , appears at above $280{ }^{\circ} \mathrm{C}$, but for the rest it can be observed at $140{ }^{\circ} \mathrm{C}$. Ion $41 \mathrm{~m} / z$ comes from $\mathrm{CH}_{2}=\mathrm{CHCH}_{2}$ group and is typical for methacrylate derivatives (Fig. 9).

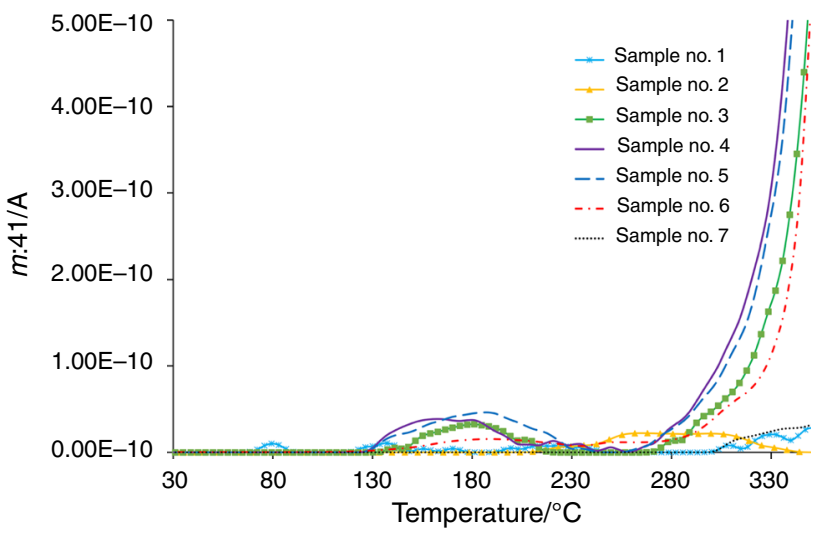

Fig. 9 Mass spectroscopy curves for ion 41

Looking at this graph, one can see same differences for these samples as it was for ion 15 . The data obtained from mass spectroscopy completely confirm the conclusions based on thermal studies. As the concentration of added photoluminescent dopant increases, the polymerization process is slightly disturbed, but samples no. 1 and 2 are completely suitable for further heat treatment and attempt to draw polymer optical fibers from them.

\section{Conclusions}

For this study, doped polymers that exhibit photoluminescent properties were prepared. Based on data obtained by thermogravimetric studies, it can be clearly stated that the polymerization process is slightly disturbed by the addition of the dopant, which can be observed as a change in the stability of the polymer matrix. The best thermal stability is observed for samples with 0.1 and 0.2 mass\% dopant concentrations. These samples have the lowest mass loss at the temperature of $220^{\circ} \mathrm{C}(0.8-0.9$ mass \%), and the initial decomposition temperature of this samples starts at $250{ }^{\circ} \mathrm{C}$ (with the mass loss 1.4-1.5 mass\%). Taking into account that polymer optical fibers are drawn in the temperature of about $220{ }^{\circ} \mathrm{C}$, for samples no. 1 and 2 drawing process can be done. Also, at this temperature for these samples, mass spectroscopy did not detect ions, indicating the initiation of thermal decomposition (ion 15). The fibers obtained in this way can be used for the optical fiber sensing applications.

Open Access This article is distributed under the terms of the Creative Commons Attribution 4.0 International License (http://creative commons.org/licenses/by/4.0/), which permits unrestricted use, distribution, and reproduction in any medium, provided you give appropriate credit to the original author(s) and the source, provide a link to the Creative Commons license, and indicate if changes were made. 


\section{References}

1. Kim DY, Cho HN, Kim CY. Blue light emitting polymers. Prog Polym Sci. 2000;11:4450-7.

2. Hong JM, Cho HN, Kim DY, Kim CY. Synthesis and luminescence studies of poly(fluorenylene ethynylene)s. Synth Met. 1999;102:933-4.

3. Vollath D, Szabo DV, Schlabach S. Oxide/polymer nanocomposites as new luminescent materials. J Nanopart Res. 2004;6:181-91.

4. Chen SH, Ando M, Murase N. Synthesis and photoluminescence of bright water-soluble CdSe/ZnS quantum dots overcoated by hybrid organic shell. Mater Lett. 2011;65:3146-9.

5. Tang J, Wang Y, Yu D, Zhou Z, Wang C, Yang B. Photoluminescence properties of an oligo-phenylkene vinylene derivative doped in polymer/silica hybrid sol-gel matrix. Mater Lett. 2001;50:371-4.

6. Zhang C, Lin J. Defect-related luminescent materials: synthesis, emission properties and applications. Chem Soc Rev. 2012;41:7938-61.

7. Museur L, Gorbovyi P, Traore M, Kanaev A, Rozes L, Sanchez C. Luminescence properties of $\mathrm{pHEMA}-\mathrm{TiO}_{2}$ gels based hybrids materials. J Lumin. 2012;132:1192-9.

8. Hu Y, Zhang Y, Gao J. Strong electroluminescence from polymer films with heavily quenched photoluminescence. Adv Mater. 2006;18:2880-3.

9. Mori T, Kijima M. Synthesis and electroluminescence properties of carbazole-containing 2,6-naphthalene-based conjugated polymers. Eur Polym J. 2009;45:1149-57.

10. Montalti M, Dolci LS, Prodi L, Zaccheroni N, Stuart MCA, van Bommel KJC. Energy transfer from a fluorescent hydrogel to a hosted fluorophore. Langmuir. 2006;22:2299-303.

11. Tomasulo M, Kaanumal SL, Sortino S, Raymo FM. Synthesis and properties of benzophenone-spiropyran and naphthalenespiropyran conjugates. J Org Chem. 2007;72:595-605.

12. Huang F, Zhang Y, Liu MS, Cheng YJ, Jen AKY. High-efficiency and color stable blue-light-emitting polymers and devices. Adv Funct Mater. 2007;17:3808-15.

13. Mergo P, Gil M, Skorupski K, Klimek J, Wójcik G, Pędzisz J, Kopec J, Poturaj K, Czyzewska L, Walewski A, Gorgol A. Low loss poly(methyl methacrylate) useful in polymer optical fibers technology. Photonics Lett Pol. 2013;5:170-2.
14. Arrue J, Jimenez F, Ayesta I, Illarramendi MA, Joseba Z. Polymer-optical-fiber lasers and amplifiers doped with organic dyes. Polymers. 2011;3:1162-80.

15. Tagaya A, Koike Y, Kinoshita T, Nihei E, Yamamoto T, Sasaki K. Polymer optical fiber amplifier. Appl Phys Lett. 1993;53:883-4.

16. Liang H, Zheng Z, Li Z, Hu J, Chen B, Zhao H, Zhang Q, Ming $\mathrm{H}$. Fabrication and amplification of rhodamine B-doped step-index polymer optical fiber. J Appl Polym Sci. 2014;93:681-5.

17. Peng GD, Xiong Z, Chu PL. Fluorescence decay and recovery in organic dye-doped polymer optical fibers. J Lightwave Technol. 1998;12:2365-72.

18. Peng GD, Xiong Z, Chu PL. Photosensitivity and gratings in dyedoped polymer optical fibers. Opt Fiber Technol. 1999;2:242-51.

19. Koeppen C, Yamada S, Jiang S, Garito AF, Dalton LR. Rareearth organic complexes for amplification in polymer optical fibers and waveguides. J Opt Soc Am B. 1997;14:155-62.

20. Zheng Z, Liang H, Ming H, Zhang Q, Xie J. Optical transition probability of the $\mathrm{Er}^{3+}$ ion in $\operatorname{Er}(\mathrm{DBM})_{3}$ phen-doped poly(methyl methacrylate). Opt Commun. 2004;233:149-53.

21. Podkościelna B, Gawdzik B. Influence of diluent compositions on the porous structure of methacrylate derivatives of aromatic diols and divinylbenzene. Appl Surf Sci. 2010;256:2462-7.

22. Podkościelna B. New photoluminescent copolymers of naphthalene-2,7-diol dimethacrylate and $N$-vinyl-2-pyrrolidone: synthesis, characterisation and properties. J Therm Anal Calorim. 2014;116:785-93.

23. Podkościelna B, Sobiesiak M. Characteristics of thermal behavior of photoluminescent copolymers studied by the TG/DTG/FTIR coupled method. J Therm Anal Calorim. 2017;127:625-31.

24. Ota K, Kikuchi S, Kopel HM, Thanos ChE, Nakamura RM. Effect of light exposure time on the depth of curing in various composite resin systems. Pediatr Dent. 1985;7:19-22.

25. Podkościelna B, Lipke A, Gawdzik B, Majdan M. Synthesis, characterization and luminescent properties of new copolymers of dimethacrylate derivatives of naphthalene-2,7-diol. Polym Adv Technol. 2015;26:176-81.

26. Zielinski W, Rajca A. Metody spektroskopowe i ich zastosowanie do identyfikacji związków organicznych. Warszawa: Wydawnictwo Naukowo Techniczne; 2000.

Publisher's Note Springer Nature remains neutral with regard to jurisdictional claims in published maps and institutional affiliations. 